\title{
Possible Risk Factors for Candida Esophagitis in Immunocompetent Individuals
}

\author{
Yousef Nassar $^{\mathrm{a}, \mathrm{d}}$, Tony Eljabbour ${ }^{\mathrm{b}}$, Hwajeong Lee $^{\mathrm{b}}$, Asra Batool $^{\mathrm{c}}$
}

\begin{abstract}
Background: Candida esophagitis (CE) is a condition typically diagnosed in patients who are immunocompromised. Risk factors leading to the development of $\mathrm{CE}$ in immunocompetent patients have not been entirely elucidated. This study set out to identify risk factors associated with the development of CE in immunocompetent patients.

Methods: This study was a single-center retrospective chart review. Patients diagnosed with CE confirmed by endoscopic biopsy or brushings at our hospital between 2007 and 2017 were reviewed. The medical histories, endoscopy reports and pathology results were noted. Abdominal pain, heartburn, dysphagia and odynophagia were the common indications for endoscopy. A total of 241 patients were identified as having been diagnosed with $\mathrm{CE}$ by endoscopic brushing or biopsy. Of these patients, 161 were excluded due to the presence of immunocompromising and 80 patients were included who had no underlying immunocompromising conditions.
\end{abstract}

Results: Eighty patients with CE satisfied the inclusion criteria. The mean age of patients at the time of diagnosis was 39.8 years old (95\% CI: 34.9 - 44.7). The incidences in men and women were similar in this study (49\% women and $51 \%$ men). Of these patients, 56 $(70 \%)(95 \%$ CI: $59-80 \%$; $P<0.005)$ were taking proton pump inhibitors (PPIs). Fifteen patients (19\%) had a previous upper endoscopy with evidence of reflux esophagitis, and they were all treated with PPIs and subsequently found to have $\mathrm{CE}$ on repeat upper endoscopy with a mean of 21.6 months of PPI treatment. There were $16(20 \%)$ patients without any attributable risk factor and were completely healthy.

Conclusions: CE is an opportunistic infection typically seen in immunocompromised. We report incidence of $\mathrm{CE}$ in immunocompetent

Manuscript submitted April 9, 2018, accepted April 20, 2018

aDepartment of Medicine, Albany Medical Center, 43 New Scotland Ave., Albany, NY 12208, USA

bDepartment of Pathology, Albany Medical Center, 43 New Scotland Ave., Albany, NY 12208, USA

'Division of Gastroenterology, Department of Medicine, Albany Medical Center, 43 New Scotland Ave., Albany, NY 12208, USA

${ }^{\mathrm{d} C}$ Corresponding Author: Yousef Nassar, Department of Medicine, Albany Medical Center, 43 New Scotland Ave., Albany, NY 12208, USA.

Email: nassary@amc.edu

doi: https://doi.org/10.14740/gr1019w patients. In our cohort of immunocompetent patients, PPI use was the most common risk factor associated with the development of CE. This could be related to hypochlorhydria resulting from PPI use. However, the cause remains unclear in some patients.

Keywords: Candida esophagitis; Healthy; Esophageal candidiasis; Proton pump inhibitors

\section{Introduction}

Candida esophagitis (CE) is the most common infection of the esophagus [1]. It is predominantly due to Candida albicans, although non-albicans species such as Candida glabrata and Candida tropicalis are increasingly prevalent [2]. C. albicans is a gastrointestinal commensal that colonizes the esophagus in up to $20 \%$ of people [3]. In addition to causing local mucosal membrane infections of the esophagus, oropharynx and vagina, Candida spp., may also lead to severe systemic infection such as bacteremia [2].

Patients with CE typically present with dysphagia and odynophagia (pain with swallowing) that can often be pinpointed to a specific retrosternal area [4]. In immunosuppressed patients (such as those with acquired immunodeficiency syndrome or "AIDS"), oral "thrush" often occurs concurrently. The presence of oropharyngeal candidiasis in a patient with esophageal symptoms can assist with diagnosis, although many patients with CE may be asymptomatic [5, 6].

A definitive diagnosis of CE can be established by endoscopy, with visible white mucosal plaque-like lesions present (Fig. 1). Endoscopic brushing or biopsy will identify yeasts and pseudohyphae that invade mucosal cells, while culture will confirm candida species [2] (Figs. 2 and 3). A cost-effective alternative to endoscopy recommended by the Infectious Diseases Society of America is a diagnostic trial of antifungal therapy [2]. Most patients with CE will experience improvement or resolution of symptoms within 7 days of commencing fluconazole orally. Patients who fail to respond need further investigation. CE may still be diagnosed on endoscopy, but might be caused by candida species or strains that are not susceptible to fluconazole [4, 7]. Among the differential diagnoses are viral infection (cytomegalovirus or herpes simplex virus, noting that these may co-infect with Candida spp.,), medication-associated esophagitis (e.g. tetracyclines, non-steroidal anti-inflammatory drugs), and inflammatory conditions 


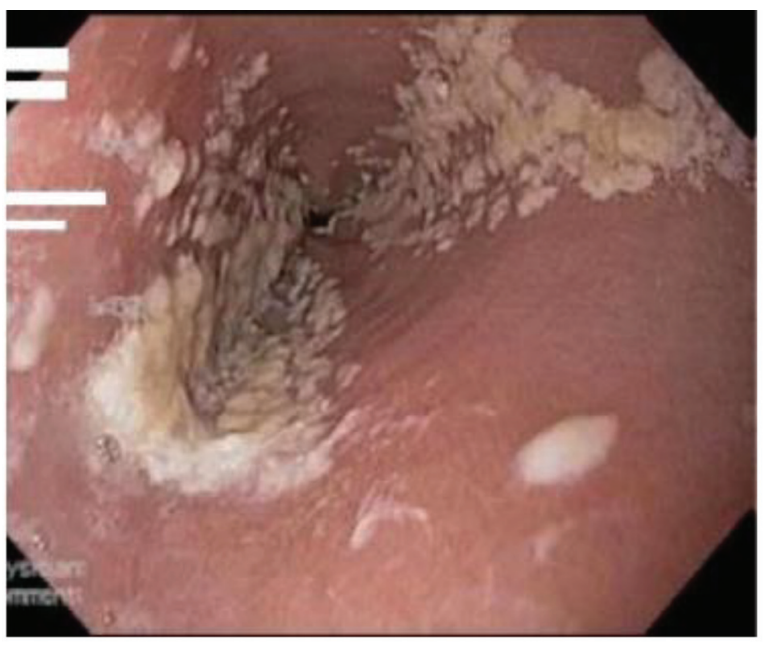

Figure 1. Candida esophagitis in one of the study patients.

(such as eosinophilic esophagitis) [8].

$\mathrm{CE}$ is considered an opportunistic infection, typically developing in individuals who are immunocompromised due to underlying medical conditions or pharmacotherapy [4]. It occurs in association with human immunodeficiency virus (HIV) infection (it is considered an AIDS-defining illness), cancer (most notably hematological malignancies), diabetes mellitus and congenital immune deficiencies. It can also develop in patients receiving immunosuppressing drugs such as long-term oral corticosteroids and cytotoxic agents [4]. In one very large cohort $(\mathrm{n}=80,219)$, the prevalence of CE amongst patients undergoing endoscopy was $1.7 \%$ overall, but $9.8 \%$ in patients with HIV infection [9].

As outlined above, a number of risk factors for $\mathrm{CE}$ have been identified that relate to impaired immunity of the host. CE can also occur in patients with an intact immune system. In this

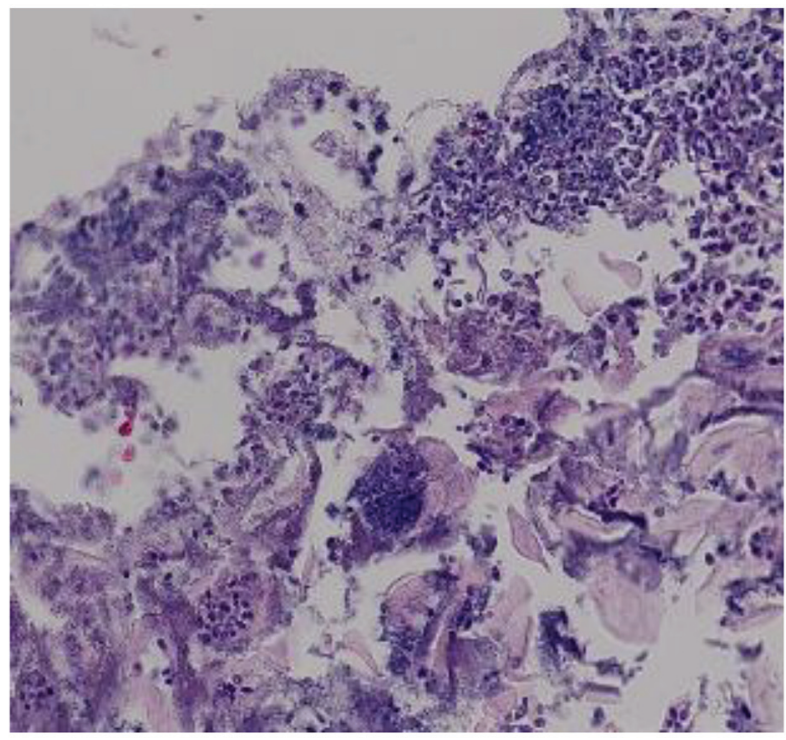

Figure 2. Hematoxylin and eosin stain of candida esophagitis biopsy in a study patient. Magnification $\times 200$.

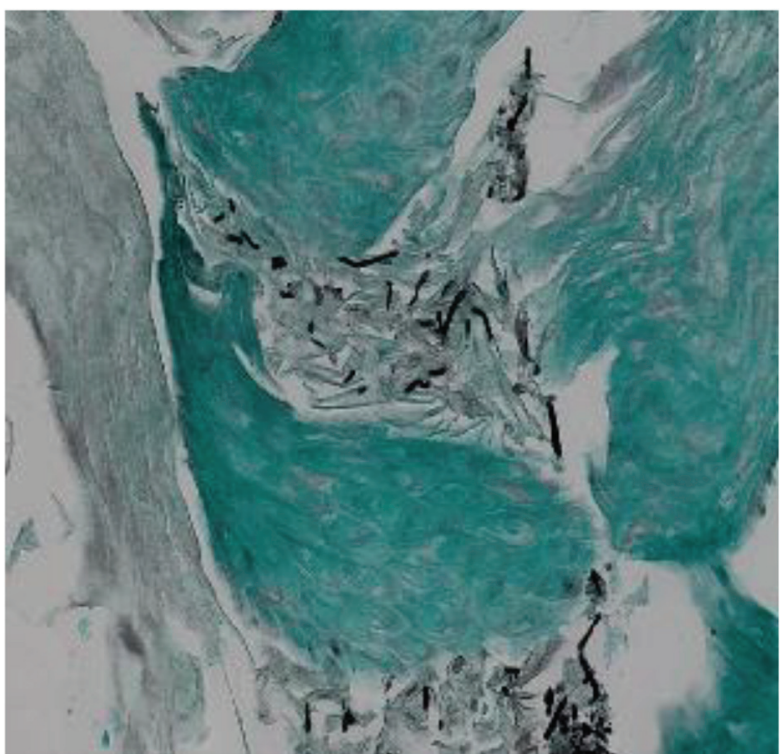

Figure 3. Methenamine silver stain of candida esophagitis biopsy in a study patient. Pseudohyphae appear black in this stain. Magnification $\times 200$.

descriptive study, possible risk factors for development of CE were explored among immunocompetent patients diagnosed with $\mathrm{CE}$ at a single tertiary care medical facility.

\section{Materials and Methods}

All inpatient and outpatients $>5$ years of age diagnosed with $\mathrm{CE}$ at Albany Medical Center, a tertiary care hospital in New York State between 1 January 2007 and 1 January 2017, were reviewed to assess eligibility for study participation. The patients were identified via records of pathological specimens with diagnosis of CE made using endoscopic brushing or biopsies. Medical records were reviewed to determine eligibility for inclusion, defined as an endoscopically (brushing or biopsy) confirmed diagnosis of CE presenting in an individual without evidence of immune system compromise. Patients were excluded if they had an underlying immunocompromising condition(s) documented at the time of CE diagnosis, defined as HIV/AIDS, any malignancy, transplantation or diabetes mellitus. They were also excluded if they took immunosuppressive medications such as long term corticosteroid, chemotherapy or immunomodulators at the time of diagnosis of CE. Only those patients with complete medical history information were included.

Medical records were reviewed for each patient eligible for study participation. Information sought included demographic details (e.g. gender, age at diagnosis), comorbid conditions and active medications at the time of diagnosis of CE. Laboratory data were reviewed to identify pathology findings from biopsies taken from the brush border or esophageal mucosa. Earlier endoscopy results were reviewed for all participants who had an endoscopy prior to diagnosis of CE. Approval from the Institutional Review Board was obtained prior to initiating the 
Table 1. Comorbidities and Endoscopic Findings in the Study Population $(n=80)$

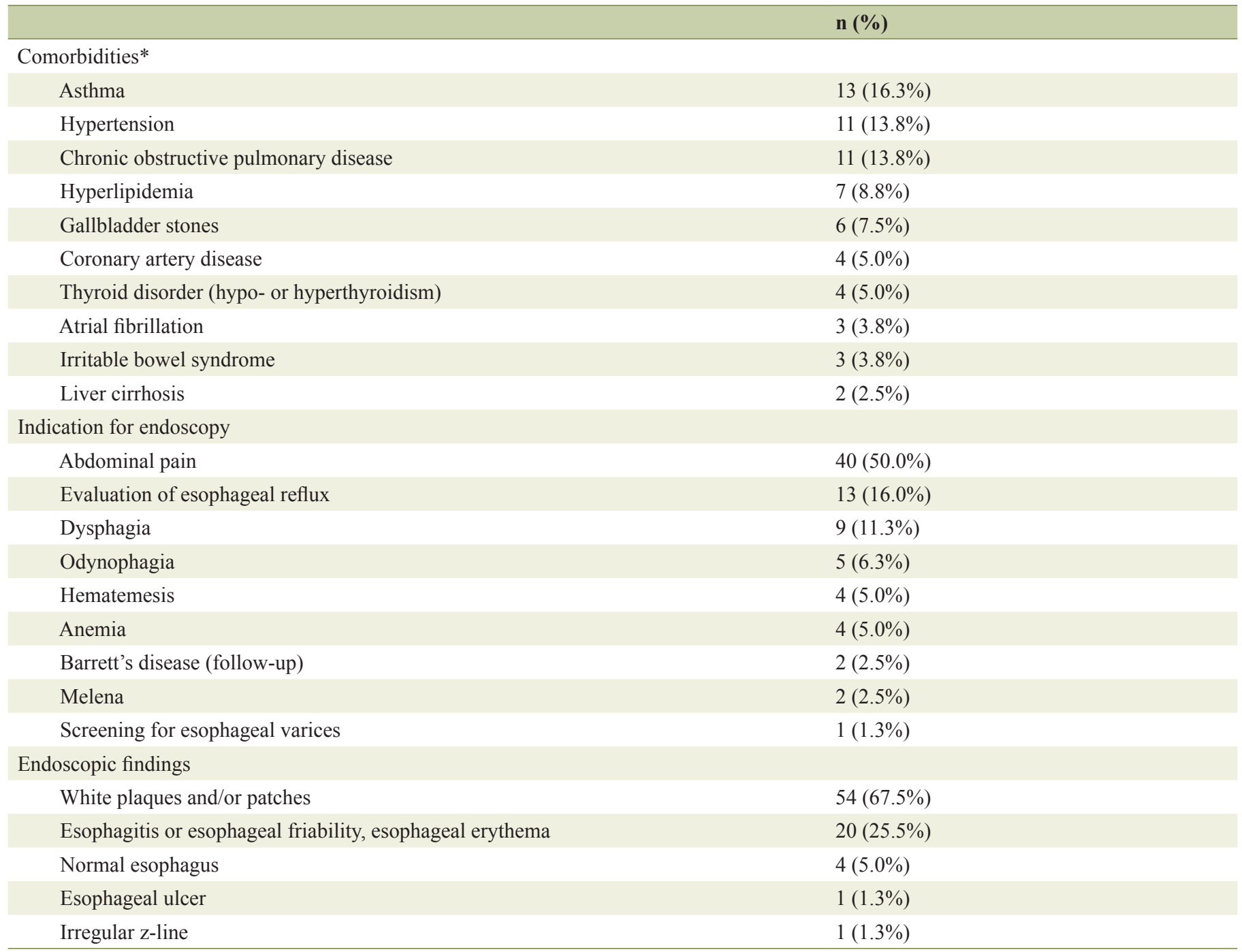

study.

\section{Results}

A total of 241 patients were identified as having been diagnosed with $\mathrm{CE}$ at Albany Medical Center over the 10-year study period. Two-thirds $(66.8 \%)$ of these $(161 / 241)$ were deemed immunocompromised and were therefore excluded from further study. The 80 remaining patients were classified as immunocompetent and were included in the study proper.

The 80 participants ( 39 males $/ 41$ females) had a mean age of 40 years (standard deviation \pm 22 years). Comorbidities at the time of CE diagnosis featured chronic respiratory conditions (asthma and chronic obstructive airways disease), which were experienced by $30 \%(24 / 80)$ of patients. Hypertension was also fairly common affecting $13.8 \%(11 / 80)$ participants (Table 1).

The indications for endoscopy and lead to the diagnosis of $\mathrm{CE}$ varied (Table 1). The most common indication was abdominal pain, which affected $50 \%$ of all participants (40/80). Less frequent triggers included symptomatic gastroesophageal reflux $(13 / 80(16.3 \%))$, dysphagia $(9 / 80(11.3 \%))$ and odynophagia $(5 / 80(6.3 \%))$ (Table 1$)$. Other triggers for endoscopy occurred at a rate of $5 \%$ or less being hematemesis, anemia, Barrett's esophagus (routine follow-up), screening for esophageal varices and melena.

Endoscopic findings of patients at the time of diagnosis of CE were noted (Table 1). Two-thirds $(67.5 \%)$ of patients had white plaques or patches (54/80) and 25\% had esophagitis, esophageal erythema and/or mucosal friability (20/80). Occurring in only one patient each were esophageal ulcer $(1.3 \%)$ and irregular z-line (1.3\%). No abnormalities were seen in four patients $(5.0 \%)$.

Three-quarters (73.8\%) of study participants were taking proton pump inhibitors (PPIs) at the time of diagnosis (59/80) and one-fifth $(18.9 \%)$ were using inhaled corticosteroids $(15 / 80)$. No identifiable risk factors were found in $16(20 \%)$ 
Table 2. Clinical Factors That Might Predispose to CE in the Study Population $(n=80)$

\begin{tabular}{ll} 
& n (\%) \\
\hline Proton pump inhibitor & $59(73.8 \%)$ \\
Corticosteroids (inhaled) & $15(18.9 \%)$ \\
No risk factors identified & $16(20.0 \%)$ \\
$\begin{array}{l}\text { Gastroesophageal reflux } \\
\text { Patients with previous endoscopies prior to CE } \\
\text { diagnosis with findings of reflux esophagitis }\end{array}$ & $50(62.8 \%)$ \\
$\begin{array}{l}\text { Patients who were started on a proton pump } \\
\text { inhibitor therapy at the time of diagnosis of reflux } \\
\text { esophagitis (15 patients with previous endoscopy) }\end{array}$ & $15(100 \%)$ \\
\hline
\end{tabular}

of our study patients and were otherwise healthy at the time of diagnosis (Table 2).

Fifteen of the 80 patients $(18.8 \%)$ had undergone an endoscopy prior to the endoscopy that resulted in the diagnosis of CE. Review of the biopsy results indicated that none of these had CE diagnosed on the initial endoscopy. However, all 15 patients $(100 \%)$ were found to have reflux esophagitis and were subsequently started or continued on PPIs at an increased dose (Table 2). The mean time between initial and follow-up endoscopy was 20.7 (standard deviation 19.02) months. The number of patients who were found to have reflux esophagitis at the time of diagnosis of CE was 19/80 (24\%).

\section{Discussion}

In this cohort of immunocompetent patients, our data suggest that use of PPIs was associated with development of CE in immunocompetent patients. Three-quarters (74\%) of all participants were found to be taking a PPI at the time of diagnosis. Further, a subset of 15 patients was endoscopically confirmed as developing $\mathrm{CE}$ subsequent to initiation or dose escalation of the acid suppressant. PPIs reduce gastric acid secretion resulting in a relative or actual state of achlorhydria [10]. This may increase the pathogenicity of $C$. albicans by altering the yeast from a rounded form to the pathogenic hyphal form, creating an environment more suitable for infection [11]. An overgrowth of $C$. albicans in the gastric lumen has been result with an elevation of $\mathrm{pH}$ post-vagotomy [12].

Fifteen (18.8\%) participants were documented as being prescribed an inhaled corticosteroid at the time of CE diagnosis. Inhaled corticosteroids are well documented precipitants of oropharyngeal candidiasis. However, the link between inhaled corticosteroids and esophageal candidiasis is largely confined to case reports. In one case, a 70-year-old female developed $\mathrm{CE}$ after commencing inhaled corticosteroids for treatment of asthma [13], without any evidence of other underlying immunosuppression.

The proportion of participants with asthma (16.3\%) or chronic obstructive pulmonary disease $(13.8 \%)$ exceeds that in the general population. However, it may well be the episodic use of systemic corticosteroids and broad-spectrum antimi- crobials to treat exacerbations that precipitates CE rather than the disease process itself [14-16]. It has also been described in some patients with disorders of esophageal motility [1]. Onefifth of patients with CE (16/80) had no identifiable risk factors, which is similar to that seen in other studies [17].

The mean age of the patients with CE in this study was 40 years. This is somewhat surprising given that old age has been previously identified as a risk factor for its development [9], perhaps due to factors such as decline in epithelial cell immunity. In this study, both genders were found to be equally affected by $\mathrm{CE}$, which is similar to what has been observed in previous studies [17].

In this study, the most common complaint leading to endoscopy and diagnosis of CE was abdominal pain, which occurred in $50 \%$ of patients. Only a minority had symptoms of dysphagia (11.3\%) and odynophagia (6.3\%) which were previously reported as typical presenting symptoms of CE. For most patients, CE was an incidental finding, found on endoscopy while investigating other complaints.

This study did unfortunately have some limitations. Being a relatively rare finding, we had a limited number of study patients. Comparing to controls would be difficult in this case given that PPIs are very commonly prescribed medications and only a small fraction of those patients develop CE. Further study of CE and the association with acid suppressing medication may provide more information on this finding in otherwise healthy patients.

\section{Conflict of Interest}

There was no conflict of interest to disclose for any of the authors involved in this study.

\section{Funding}

There was no source of funding for this study.

\section{References}

1. O'Rourke A. Infective oesophagitis: epidemiology, cause, diagnosis and treatment options. Curr Opin Otolaryngol Head Neck Surg. 2015;23(6):459-463.

2. Pappas PG, Kauffman CA, Andes DR, Clancy CJ, Marr KA, Ostrosky-Zeichner L, Reboli AC, et al. Clinical practice guideline for the management of candidiasis: 2016 update by the infectious diseases society of America. Clin Infect Dis. 2016;62(4):e1-50.

3. Vermeersch B, Rysselaere M, Dekeyser K, Rasquin K, De Vos M, Elewaut A, Barbier F. Fungal colonization of the esophagus. Am J Gastroenterol. 1989;84(9):1079-1083.

4. Rosolowski M, Kierzkiewicz M. Etiology, diagnosis and treatment of infectious esophagitis. Prz Gastroenterol. 2013;8(6):333-337.

5. Vazquez JA. Optimal management of oropharyngeal and esophageal candidiasis in patients living with HIV infec- 
tion. HIV AIDS (Auckl). 2010;2:89-101.

6. Samonis G, Skordilis P, Maraki S, Datseris G, Toloudis P, Chatzinikolaou I, Georgoulias V, et al. Oropharyngeal candidiasis as a marker for esophageal candidiasis in patients with cancer. Clin Infect Dis. 1998;27(2):283-286.

7. Kakati B, Kotwal A, Biswas D, Sahu S. Fluconazole resistant candida oesophagitis in immunocompetent patients: Is empirical therapy justifiable? J Clin Diagn Res. 2015;9(12):16-18

8. Geagea A, Cellier C. Scope of drug-induced, infectious and allergic esophageal injury. Curr Opin Gastroenterol. 2008;24(4):496-501.

9. Takahashi Y, Nagata N, Shimbo T, Nishijima T, Watanabe $\mathrm{K}$, Aoki T, Sekine K, et al. Long-term trends in esophageal candidiasis prevalence and associated risk factors with or without HIV infection: lessons from an endoscopic study of 80,219 patients. PLoS One. 2015;10(7):e0133589.

10. Corleto VD, Festa S, Di Giulio E, Annibale B. Proton pump inhibitor therapy and potential long-term harm. Curr Opin Endocrinol Diabetes Obes. 2014;21(1):3-8.

11. Vylkova S, Carman AJ, Danhof HA, Collette JR, Zhou H, Lorenz MC. The fungal pathogen Candida albicans au- toinduces hyphal morphogenesis by raising extracellular pH. MBio. 2011;2(3):e00055-00011.

12. Brooks JR, Smith HF, Pease FB, Jr. Bacteriology of the stomach immediately following vagotomy: the growth of Candida albicans. Ann Surg. 1974;179(6):859-862.

13. Simon MR, Houser WL, Smith KA, Long PM. Esophageal candidiasis as a complication of inhaled corticosteroids. Ann Allergy Asthma Immunol. 1997;79(4):333338.

14. Weerasuriya N, Snape J. A study of candida esophagitis in elderly patients attending a district general hospital in the UK. Dis Esophagus. 2006;19(3):189-192.

15. Kondo T, Terada K. Candida Esophagitis. N Engl J Med. 2017;376(16):1574.

16. Choi JH, Lee CG, Lim YJ, Kang HW, Lim CY, Choi JS. Prevalence and risk factors of esophageal candidiasis in healthy individuals: a single center experience in Korea. Yonsei Med J. 2013;54(1):160-165.

17. Kliemann DA, Pasqualotto AC, Falavigna M, Giaretta T, Severo LC. Candida esophagitis: species distribution and risk factors for infection. Rev Inst Med Trop Sao Paulo. 2008;50(5):261-263. 